SHORT REPORT

\title{
Visual prognosis after indirect traumatic optic neuropathy
}

\author{
A Carta, L Ferrigno, M Salvo, S Bianchi-Marzoli, A Boschi, F Carta
}

J Neurol Neurosurg Psychiatry 2003;74:246-248

Objective: To investigate a possible correlation between final visual acuity and the presence at baseline of various systemic and local (orbital/ocular) signs in patients affected by indirect traumatic optic neuropathy.

Methods: 35 cases of traumatic optic neuropathy were examined retrospectively and 13 variables were tested. Univariate analysis with "no recovery of visual acuity" as the primary outcome was performed. Relative risk (RR) and 95\% confidence intervals (Cl) were calculated. Fisher's exact test was used for two variables to test differences between proportions.

Results: Four variables showed a significantly increased risk for no recovery of visual acuity: presence of blood within the posterior ethmoidal cells $(R R=2.25,95 \% \mathrm{Cl}$ 1.25 to 4.04 ); age over 40 years ( $R R=1.79,1.07$ to 2.99); loss of consciousness associated with traumatic optic neuropathy ( $R R=2.21,1.17$ to 4.16$)$; and absence of recovery after 48 hours of steroid treatment $(p<0.01$, Fisher's exact test). Recovery documented at the first follow up visit after treatment was significantly associated with recovery at the last follow up visit $(p<0.01$, Fisher's exact test).

Conclusions: These four negative prognostic signs in patients affected by traumatic optic neuropathy may be useful in predicting the visual outcome in patients developing visual loss after head trauma and in deciding on the need for surgical treatment.

ndirect damage to the optic nerve is the most common form of traumatic optic neuropathy, occurring in $0.5-5 \%$ of all closed head trauma cases. ${ }^{12}$ As well as their other neurological problems, such patients may suffer from extremely severe visual impairment. Although the degree of visual loss after indirect traumatic optic neuropathy may be quite variable, approximately $50 \%$ of patients are left with "light perception" or "no light perception" vision, making traumatic optic neuropathy a significant cause of permanent visual loss. ${ }^{3-5}$ At present, there is no proven form of treatment for this condition and there is controversy over its optimal management. Comparative data from the international optic nerve trauma study (IONTS) group ${ }^{6}$ show that "neither corticosteroid therapy nor optic canal decompression are the gold standard for treatment of traumatic optic neuropathy."

Cook et al, in a meta-analysis, ${ }^{7}$ reported that recovery of vision in patients treated with megadosage steroids or surgical decompression of the optic canal was significantly better than recovery in patients receiving no treatment. The same conclusion, however, has not been supported by the IONTS study, which found no significant difference in final visual acuity between treated and untreated patients. As there are no controlled guidelines for a correct management, patients affected by traumatic optic neuropathy remain a clinical dilemma. A recent uncontrolled case series ${ }^{8}$ reported that visual recovery occurring shortly after trauma may not be permanent at long term follow up, with progressive deterioration of visual function.
The identification of prognostic signs during the hospital admission of such patients has important implications, as such signs may be used to justify medical or surgical treatment depending on the expected outcome. ${ }^{79}$ Furthermore, the IONTS group has found that selected cases with initially negative prognostic signs may show clear benefit from treatment versus observation alone. For these reasons, we undertook a retrospective study of patients with traumatic optic neuropathy to investigate a possible correlation between final visual acuity and several systemic and local (orbital/ ocular) signs which may have prognostic importance.

\section{METHODS}

We carried out a retrospective study in four specialised hospitals. The diagnosis of traumatic optic neuropathy was made in the presence of decreased visual acuity after a history of head trauma associated with a relative afferent pupillary defect.

Cases of post-traumatic visual loss not related to optic nerve dysfunction (open globe, traumatic cataract, retinal detachment, choroidal rupture, vitreous haemorrhage) were not included in the study.

When possible, patients were investigated with visual evoked potentials (VEP) in order to confirm the optic nerve lesion. As further support for the diagnosis, a variable amount of optic atrophy was detected in each patient at the three months follow up visit. Patients with prolonged loss of consciousness (lasting more than five days) were not included in our study, as their early visual function could not be assessed.

All patients diagnosed with traumatic optic neuropathy had been admitted to hospital. In each case high resolution orbital computed tomography (CT) was done in order to rule out the presence of optic canal fractures or dislocation of bone fragments in the orbital apex. In 12 cases magnetic resonance imaging (MRI) was also done to evaluate the intraorbital and intracranial portions of the optic nerve.

All patients underwent steroid megadose treatment within 72 hours of the trauma, according to the protocol used in the third national acute spinal cord injury study. ${ }^{10}$

Each patient underwent a baseline neuro-ophthalmological examination as soon as their mental state and physical condition were adequate for visual function testing. In addition to visual acuity measurements, patients were tested for visual fields by the confrontation method.

The examination was repeated every 48 hours in the first week. After one week steroids were tapered and patients were evaluated weekly for the first month, then monthly for the following three months. After three months, the patients were assigned to variable periodic follow up visits. To be included in this study, at least three months of follow up were required.

The local and systemic variables evaluated in our study are shown in table 1 . These were included in a univariate analysis, with absence or presence of a difference between final and baseline visual acuity as the dependent variable. Recovery of vision was defined as any improvement by at least one decimal fraction of Snellen visual acuity confirmed at the last follow up visit. In order to provide a common scale of visual acuity for analysis, all data were converted into decimal fractions of Snellen visual acuity. ${ }^{11}$ Relative risks (RR) and 95\% confidence 


\begin{tabular}{|c|c|c|c|c|}
\hline $\begin{array}{l}\text { Independent } \\
\text { variable }\end{array}$ & $\begin{array}{l}\text { Number } \\
\text { of cases }\end{array}$ & $\begin{array}{l}\text { Visual acuity on } \\
\text { recovery (n (\%)) }\end{array}$ & $\mathrm{RR}$ & $(95 \% \mathrm{Cl})$ \\
\hline \multicolumn{5}{|c|}{ CT evidence of orbital haemorrhage or bone fracture (not involving the optic canal) } \\
\hline No & 14 & $5(36)$ & 1 & \\
\hline Yes & 21 & $15(71)$ & 2.00 & $(0.94$ to 4.25$)$ \\
\hline \multicolumn{5}{|c|}{$\mathrm{CT}$ or MRI evidence of bood within posterior ethmoidal cells } \\
\hline No & 21 & $8(38)$ & 1 & \\
\hline Yes & 14 & $12(86)$ & 2.25 & (1.25 to 4.04 ) \\
\hline \multicolumn{5}{|c|}{ Immediate amaurosis after trauma } \\
\hline No & 7 & $1(14)$ & 1 & \\
\hline Yes & 28 & $19(68)$ & 4.75 & $(0.76$ to 29.6$)$ \\
\hline \multicolumn{5}{|c|}{ Evidence of ocular trauma (not involving the macula) } \\
\hline No & 26 & $16(58)$ & 1 & \\
\hline Yes & 9 & $5(56)$ & 0.96 & $(0.49$ to 1.88$)$ \\
\hline \multicolumn{5}{|l|}{ Age } \\
\hline$\leqslant 40$ years & 24 & $11(46)$ & 1 & \\
\hline$>40$ years & 11 & $9(82)$ & 1.79 & (1.07 to 2.99 ) \\
\hline \multicolumn{5}{|c|}{ Severe lesions of thorax, shoulders, upper arms, or neck } \\
\hline No & 30 & $17(57)$ & 1 & \\
\hline Yes & 5 & $3(60)$ & 1.06 & $(0.48$ to 2.31$)$ \\
\hline \multicolumn{5}{|c|}{ Evidence of cerebral lesions on imaging } \\
\hline No & 24 & $13(54)$ & 1 & \\
\hline Yes & 11 & $7(64)$ & 1.17 & $(0.66$ to 2.10$)$ \\
\hline \multicolumn{5}{|c|}{ Loss of consciousness } \\
\hline No & 19 & $7(37)$ & 1 & \\
\hline Yes & 16 & $13(81)$ & 2.21 & (1.17 to 4.16$)$ \\
\hline \multicolumn{5}{|c|}{ History of vascular diseases, mitochondriopathy or migraine } \\
\hline No & 34 & $19(56)$ & - & - \\
\hline Yes & 1 & $1(100)$ & & \\
\hline \multicolumn{5}{|l|}{ Bilaterality } \\
\hline No & 33 & - & & - \\
\hline Yes & 2 & & $p>0.5^{\star}$ & \\
\hline \multicolumn{5}{|c|}{ Recovery 48 hours after start of steroid treatment } \\
\hline No & 23 & $20(87)$ & & \\
\hline Yes & 12 & 0 & $\mathrm{p}<0.01^{*}$ & \\
\hline \multicolumn{5}{|c|}{ Ophthalmoplegia associated } \\
\hline No & 30 & $18(60)$ & 1 & \\
\hline Yes & 5 & $2(40)$ & 0.67 & $(0.22$ to 2.03$)$ \\
\hline \multicolumn{5}{|c|}{ Visual field defect on confrontation test } \\
\hline$\leqslant 50 \%$ & 14 & $5(36)$ & 1 & \\
\hline$>50 \%$ & 21 & $15(71)$ & 2.00 & (0.94 to 4.25 ) \\
\hline
\end{tabular}

intervals (CI) were calculated between prognostic signs and recovery of visual acuity. Three variables were analysed by Fisher's exact test with probability $(\mathrm{p})$ value estimation.

Of a total of 47 consecutive cases of traumatic optic neuropathy observed in the four participating clinics, 35 satisfied our criteria and were included in the analysis.

\section{RESULTS}

Table 1 summarises the results of our study. Using univariate analysis with $95 \%$ confidence intervals, three variables showed a significant increased risk for no recovery of visual acuity from baseline. These were:

- the presence of blood within the posterior ethmoidal cells detected on MRI or CT (fig 1) ( $\mathrm{RR}=2.25$ for no recovery, $95 \%$ CI 1.25 to 4.04 );

- loss of consciousness occurring during trauma $(\mathrm{RR}=2.21$, 1.17 to 4.16 );

- age over 40 years ( $\mathrm{RR}=1.79,1.07$ to 2.99 ).

Another variable associated with no recovery was immediate amaurosis after trauma ( $R R=4.75,0.76$ to 29.6), though the range of the confidence interval for this variable did not reach statistical significance.

Using Fisher's exact test, a fourth variable was found to be associated with poor recovery. This was the absence of improvement after 48 hours of steroid treatment $(p<0.01)$.

Bilateral involvement and concomitant systemic vascular disease were not correlated with final visual acuity $(p>0.5)$. In contrast, the presence of visual recovery at the 48 hour visit was significantly associated with recovery of visual acuity at the last follow up examination $(p<0.01)$. Twenty three of the 35 patients $(87 \%)$ with no improvement after 48 hours showed no improvement of visual acuity at the last follow up visit.

The mean decimal fraction of Snellen visual acuity calculated as the visual recovery in our population was 0.393 (range 0.05 to $0.95, \mathrm{SD}=0.25$ ).

\section{DISCUSSION}

The pathogenesis of traumatic optic neuropathy is unclear, with several possible mechanisms responsible for visual loss. The optimal treatment for this condition is also 


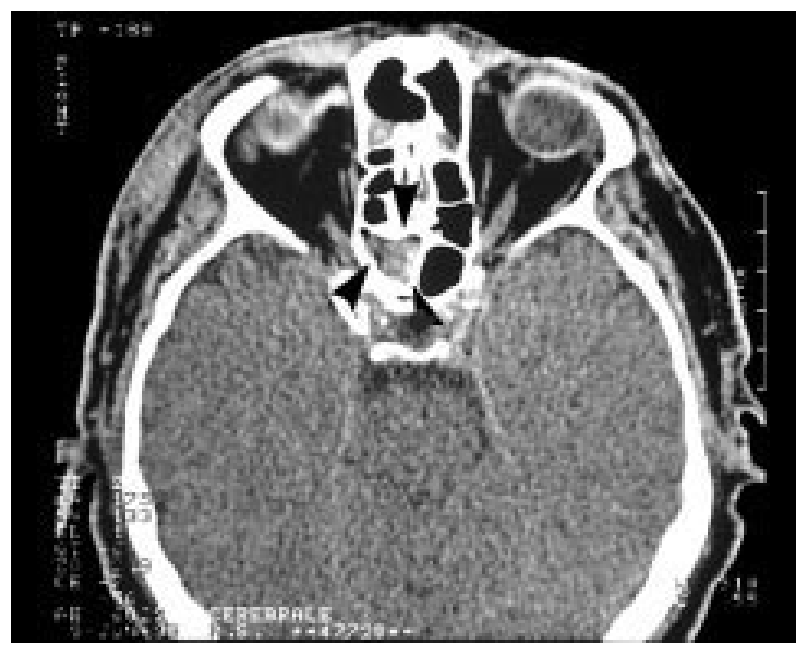

Figure 1 Cerebral computed tomography showing blood within posterior ethmoidal cells (arrowheads) on an axial plane. The patient was a white male, 52 years old, involved in a car accident with transient loss of consciousness. Visual acuity was light perception at the first visit, 12 hours after trauma. One year later visual acuity was unchanged and severe optic atrophy was clearly evident on fundoscopic examination.

controversial, ${ }^{12}$ and the management tends to be on the basis of personal preference. As suggested by Levin et al, ${ }^{6}$ it is possible that selected patients will benefit from either corticosteroids or surgery. For this reason, assessment of visual prognostic signs during the hospital admission of such patients has relevant clinical implications. Our study suggests that four different variables are related to a poor outcome in patients with traumatic optic neuropathy: blood in the posterior ethmoid cells, loss of consciousness, age over 40, and absence of improvement after two days of steroid treatment.

Previous laser interferometric studies have shown that forces applied to the frontal bone are transferred and concentrated in the optic canal region. ${ }^{13}$ Histopathological studies of patients with indirect injury to the optic nerve have been consistent with localisation of the lesion to this area. ${ }^{14}$ Bleeding within the posterior ethmoidal cells presumably reflects a greater amount of energy applied to this region and it should therefore make sense that this sign is associated with a worse visual prognosis. Similarly, as ethmoidal bleeding reflects the local severity of trauma, it could also account for the fact that loss of consciousness is associated with a reduced likelihood of visual recovery.

Age was another variable associated with a poor outcome. We speculate that recovery of vision in such patients may be impaired by age related axonal lipoperoxidation and membrane hydrolysis occurring after the trauma. This hypothesis is supported by recent studies on mammals in which brain aging is associated with a gene expression profile indicative of oxidative stress and reduced neurotrophic support in the neocortex. ${ }^{15} 16$

In our study, $87 \%$ of our patients who improved from baseline performance experienced visual recovery within 48 hours of the start of steroid treatment. For this reason, traumatic optic neuropathy cases with severe visual loss (acuity less than 20/200) and no improvement after 48 hours of steroid therapy may be considered candidates for optic canal decompression. Patients with negative prognostic signs (visual acuity less than 20/200, blood in the posterior ethmoidal cells, age over 40 , and loss of consciousness during trauma) may benefit from surgical treatment. Surgical decompression of the optic canal has a risk of several complications, but the results of our study suggest a rational basis for such surgery.
The optimal treatment and its timing for traumatic optic neuropathy need to be evaluated in a prospective study. In our study, as in many previous ones, the primary outcome was based entirely on visual acuity, whereas some patients may recover useful visual fields but with poor visual acuity. The lack of visual field data is related to the inability of patients to undertake automated or cinetic perimetry at baseline (for logistic and medical reasons). For this reason we were unable to obtain standardised visual field parameters to include in the analysis as a dependent variable.

\section{Conclusions}

Despite the limitations related to a univariate analysis, the negative prognostic signs identified may have implications for the management of traumatic optic neuropathy.

\section{Authors' affiliations}

A Carta, Department of Ophthalmology, University of Parma, Parma, Italy

M Salvo, Department of Ophthalmology, University of Sassari, Sassari, Italy

S Bianchi-Marzoli, Department of Ophthalmology, University of Milan, Milan, Italy

A Boschi, Department of Ophthalmology, University of Brussels, Brussels, Belgium

L Ferrigno, Laboratory of Epidemiology and Biostatistics, Istituto

Superiore di Sanità, Rome, Italy

F Carta, Department of Opthalmology, University of Sassari, Sassari, Italy

Competing interests: none declared

Correspondence to: Dr Arturo Carta, Neuro-Ophthalmology Service, Department of Ophthalmology, University of Parma, via Gramsci 14, 43100 Parma, Italy; acarta@unipr.it

Received 8 February 2002

In revised form 31 July 2002

Accepted 4 October 2002

\section{REFERENCES}

1 Kline LB, Morawetz RB, Swaid SN. Indirect injury of the optic nerve. Neurosurgery 1984;14:756-64.

2 Kallela I, Hyrkas T, Paukku P, et al. Blindness after maxillofacial blunt trauma. J Oral Maxillofac Surg 1994;22:220-5.

3 Lessell S. Indirect optic nerve trauma. Arch Ophthalmol 1989; 107:382-6

4 Steinsapir KD, Goldberg RA. Traumatic optic neuropathy. Surv Ophthalmol 1994;38:487-516.

5 Chou PI, Sadun AA, Chen CY, et al. Clinical experiences in the management of traumatic optic neuropathy. Neuroophthalmology $1996 ; 16: 325-36$

6 Levin LA, Beck RW, Joseph MP, et al. The treatment of traumatic optic neuropathy. Ophthalmology 1999;106:1268-77.

7 Cook MW, Levin LA, Joseph MP, et al. Traumatic optic neuropathy: a meta-analysis. Arch Otolaryngol Head Neck Surg 1996;122:389-92.

8 Mariak Z, Obuchowska I, Mariak Z, et al. High-dose steroid therapy of traumatic optic neuropathy may fail to protect the optic nerve permanently. Neuroophthalmology 1999;21:255-60.

9 Levin LA, Joseph MP, Rizzo JF, et al. Optic canal decompression in indirect optic nerve trauma. Ophthalmology 1994;101:566-9.

10 Bracken MB, Shepard M, Holford TR, et al. Administration of methylprednisolone for 24 or 48 hours or tirilazad mesylate for 48 hours in the treatment of acute spinal cord injury. JAMA 1997;277:1597-604.

11 Ferris FL, Kassoff A, Bresnick GH, et al. New visual acuity charts for clinical research. Am J Ophthalmol 1982;94:91-6.

12 Mauriello JA, De Luca J, Krieger A, et al. Management of traumatic optic neuropathy - a study of 23 patients. Br J Ophthalmol 1992;76:349-52

13 Gross CE, DeKock JR, Panje WR, et al. Evidence for orbital deformation that may contribute to monocular blindness following minor frontal head trauma. J Neurosurg 1981;55:963-6.

14 Crompton MR. Visual lesions in closed head injury. Brain 1970;93:785-92.

15 Cheol-Koo L, Weindruch R, Prolla TA. Gene-expression profile of the ageing brain in mice. Nat Genet 2000;25:294-7.

16 Sohal RS, Weindruch R. Oxidative stress, caloric restriction, and aging. Science 1996;273:59-63. 